\title{
Unifocalization of the neck arteries combined with aortic arch replacement for Williams syndrome
}

\author{
Yoshiaki Yamada, MD, Masaaki Yamagishi, MD, Keisuke Shuntoh, MD, Takahisa Okano, MD, \\ Kyoko Hayashida, MD, Takeshi Shinkawa, MD, and Nobuo Kitamura, MD, Kyoto, Japan
}

$\mathrm{V}$ arious types of supravalvular aortic stenosis (SVAS) and deformities of the aorta and neck branch arteries are combined in Williams syndrome. ${ }^{1}$ Although several surgical options have been reported for SVAS, it is often difficult to obtain relief of the diffuse type of SVAS. We report an alternative surgical technique for Williams syndrome consisting of unifocalization of the neck branch arteries and straight graft replacement of the aorta.

\section{Clinical Summary}

A 16-year-old boy with an elfin face, but without mental retardation, was referred to our hospital for surgical repair. The diagnosis of SVAS was made when he was 8 years old. On admission, a systolic heart murmur and bruit were audible at the bilateral cervical area. Electrocardiography during a treadmill test revealed left ventricular hypertrophy and ischemic change. Echocardiography showed that the aortic valve was competent and the aortic anulus was $22 \mathrm{~mm}$ in diameter. Cardiac catheterization revealed severe diffuse stenosis at the ascending aorta extending to the transverse arch and narrowing at the orifice of the right brachiocephalic artery and the left common carotid artery (Figure 1). A pressure gradient of $60 \mathrm{~mm} \mathrm{Hg}$ was measured between the ascending aorta and the left ventricle. Peripheral pulmonary arterial stenosis was not detected. Although he had no symptoms, surgical intervention was planned because of left ventricular pressure overload.

The operation was performed through a median sternotomy. The aorta and the neck arteries were not narrow in appearance. Each neck artery was dissected as distally as possible. An arterial

From the Department of Pediatric Cardiovascular Surgery, Children's Research Hospital, Kyoto Prefectural University of Medicine, Kyoto, Japan.

Received for publication Aug 9, 2001; accepted for publication Aug 28, 2001.

Address for reprints: Yoshiaki Yamada, MD, Department of Pediatric Cardiovascular Surgery, Children's Research Hospital, Kyoto Prefectural University of Medicine, Kawaramachi, Hirokoji, Kamikyo-ku, Kyoto, 6028566, Japan (E-mail: yyama@diana.dti.ne.jp).

J Thorac Cardiovasc Surg 2002;123:579-80

Copyright $\odot 2002$ by The American Association for Thoracic Surgery

$0022-5223 / 2002 \$ 35.00+0 \quad \mathbf{1 2 / 5 4 / 1 1 9 8 8 1}$

doi:10.1067/mtc.2002.119881 cannula was inserted directly into the descending aorta at the level of the left pulmonary artery. Three neck branch arteries and both venae cavae were also cannulated. Moderate hypothermic cardiopulmonary bypass with selective cerebral perfusion was then established. The descending aorta was clamped a few centimeters distal to the origin of the left subclavian artery. Neck arteries were squeezed by Teflon tape with a tourniquet. After chemical cardiac arrest, the ascending aorta was divided. The medial layer of the aortic wall was markedly thickened. The thick aortic wall including the superior margin of the sinotubular junction was cut away from the sinus of Valsalva. The aortic valve was competent. As in the Brom maneuver, ${ }^{2}$ longitudinal incisions a few millimeters long were made at each sinus of Valsalva. Degenerated thick vessel walls at the ascending aorta, aortic arch, and origin of the neck branch arteries were totally resected. A 20-mm Dacron graft (Hemashield; Meadox Medicals, Oakland, NJ) was anastomosed to the proximal stump of the ascending aorta according to Brom's technique. After the stumps of the neck arteries had been incised longitudinally, adjacent neck arteries were anastomosed in side-toside fashion with an absorbable polydioxanone suture (PDS II; Ethicon, Inc, Somerville, NJ) (Figure 2). End-to-end anastomosis between the distal end of a Dacron graft and the common orifice of the unifocalized neck arteries and the distal aortic arch was completed. The postoperative course was uneventful. Postoperative 3dimensional images by helical computed tomographic scanning showed no residual stenosis in any vessel.

\section{Discussion}

Various vascular deformities of the systemic and pulmonary arteries concomitant with Williams syndrome are caused by mutations of the elastin genes. ${ }^{3}$ Aortic anomalies of the Williams syndrome are grouped into supra-aortic localized hourglass or membranous stenosis and diffuse narrowing often extending to the neck branch arteries. The surgical strategy for the localized type of SVAS has been established. Brom's technique may provide complete relief for the localized type of SVAS and restoration of normal aortic root geometry without aortic insufficiency. ${ }^{4}$ However, the conventional procedures for the diffuse narrowing type of SVAS, such as patch aortoplasty, are still no better than a palliative operation. Complete relief of diffuse SVAS, especially in which the lesion extends to the neck arteries, is obviously difficult. Residual stenosis and late restenosis are often inevitable by the conventional procedures. Replacement by a prosthetic graft with 3 branches is necessary for the diffuse-type SVAS extending to the neck branch arteries, whereas an appropriate ready-made 3-branch graft for 

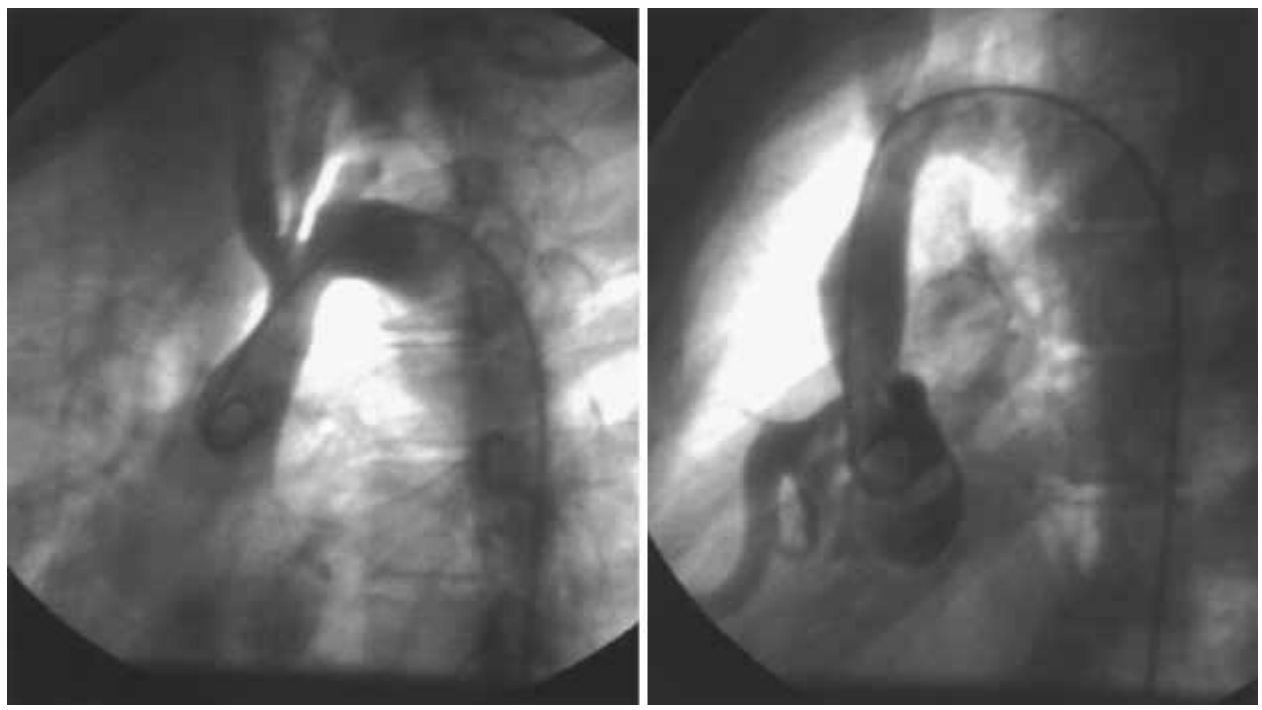

Figure 1. Aortograms demonstrated diffuse SVAS with narrowing at the orifice of right brachiocephalic artery and left common carotid artery.
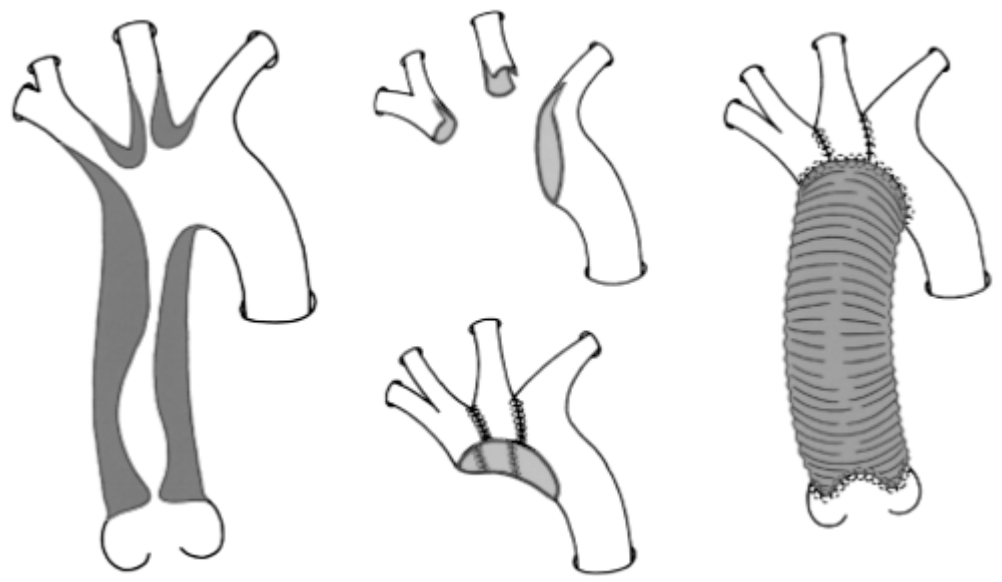

Figure 2. Schematic representation of the operative procedure. Unifocalization of the neck arteries and replacement of the aorta with a straight graft.

juvenile patients is not commercially available. Therefore, we developed an alternative technique consisting of unifocalization of the neck arteries with a unified distal aortic orifice. The proximal SVAS was reconstructed according to Brom's procedure. This innovative technique can simplify the operation by reduction of the site of anastomosis between the graft and the native arteries. If reoperation is required because of peel formation or relative narrowing with the growth, re-replacement of the graft may be easy, because a straight graft is used in this technique. This technique can also be adapted for infantile diffuse SVAS. Further investigation of the unfocalized neck arteries is needed.

\section{References}

1. Kumar A, Stalker HJ, Williams CA. Concurrence of supravalvular aortic stenosis and peripheral pulmonary stenosis in three generations of a family: a form of arterial dysplasia. Am J Med Genet. 1993;45:739-42.

2. Brom AG. Obstruction of the left ventricular outflow tract. In: Khonsari S, editor. Cardiac surgery: safeguards and pitfall in operative technique. Rockville (MD): Aspen; 1988. p. 276-80.

3. Keating MT. Genetic approaches to cardiovascular disease: supravalvular stenosis, Williams syndrome, and long-QT syndrome. Circulation. 1995:92:142-7.

4. Myers JL, Waldhausen JA, Cyran SE, Gleason MM, Weber HS, Baylen BG. Results of surgical repair of congenital supravalvular aortic stenosis. J Thorac Cardiovasc Surg. 1993;105:281-8. 